\title{
Dwarf Galaxies and Star Clusters in Tidal Tails
}

\author{
Peter M. Weilbacher, Uta Fritze-von Alvensleben \\ Universitäts-Sternwarte Göttingen, Geismarlandstr. 11, 37083 \\ Göttingen, Germany \\ Pierre-Alain Duc \\ CNRS and CEA/DSM/DAPNIA/SAp, Saclay, 91191 Gif sur Yvette \\ cedex, France
}

\begin{abstract}
We summarize the properties of tidal dwarf candidates in a sample of interacting galaxies and classify objects in tidal tails depending on their morphological appearance. New high-resolution dynamical models are needed to understand how the different structures seen in tidal tails are formed.
\end{abstract}

\section{Introduction}

First we want to stress the new definition for Tidal Dwarfs given by Weilbacher \& Duc (2001), who - following Duc et al. 2000 - define a Tidal Dwarf Galaxy (TDG) as a self-gravitating entity of dwarf-galaxy mass built from tidal material expelled during interactions. To prove that a given 'knot' in or near a tidal feature is a real TDG, one therefore has to show several properties:

- Luminosity (and therefore mass) of a dwarf galaxy.

- Association with the interacting system.

- Internal kinematics decoupled from the environment.

- Not a preexisting dwarf galaxy involved in the interaction.

- The object has to have a possible future existence to call it an 'entity'.

The luminosity and the scale length of objects that this definition allows therefore clearly separate TDGs from young or old star clusters, which do not show rotation and have lower luminosities and masses.

Typical interacting systems where gas-rich, star-forming TDGs are believed to have been found are NGC 7252 (Hibbard \& Mihos 1995), Arp 242 (Duc et al., in prep), Arp 105 (Duc et al. 1997), and Arp 245 (Duc et al. 2000). A formation sequence outlining a possible way these objects are formed has been given by Weilbacher \& Duc (2001) based on existing numerical simulations: the condensations can first appear in the gaseous or stellar component, and the other component is then pulled into the potential well. Collapsing gas could then initiate the observed star formation in these tidal dwarfs. 


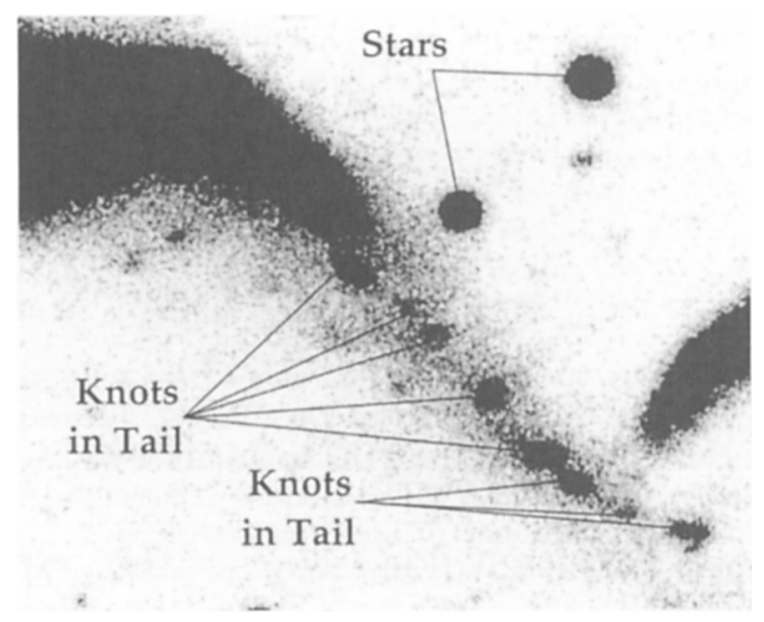

Figure 1. $\quad B$-band image of the southern tail of AM 1353-272.

\section{Summary of TDG properties}

We have analyzed a sample of 14 interacting systems (10 of them are discussed in Weilbacher et al. 2000). About 40 knots in the tidal features of these systems show properties which make them plausible TDG candidates. They are brighter than -11 mag in $M_{B}$, mostly low surface brightness, and are extended. This differentiates them from possible clusters and meets the first property listed above. They also show blue colors, and are sometimes the bluest part of the entire interacting system, which hints at active star formation. Indeed they also show emission line spectra, which enables us to measure the redshift and the metallicity in the gas phase. Several of these TDG candidates also show signs of dynamics in their 2D spectra, indicative of internal kinematics.

From comparison with evolutionary synthesis models (see Weilbacher \& Fritze-v:Alvensleben 2001) we were able to estimate burst ages and strengths. It was seen from the broad-band colors that these TDG candidates indeed experience strong bursts increasing the stellar mass by up to $20 \%$, and have young burst ages up to 20 Myrs (Weilbacher et al. 2000). Preliminary mass estimates based on optical luminosities show stellar masses of several $10^{7} \mathrm{M}_{\odot}$ or more.

An indication of 'normal' star formation in TDGs has been found by Lisenfeld et al. (2001) by the detection of CO in 8 of 11 TDGs of interacting systems in a different sample, with an efficiency similar to that in spiral galaxies.

\section{Structures in Tidal Tails}

Looking at images of tidal features one can see different structures in the tails (see also Hibbard 2001):

1. TDG(s): One or two tidal dwarfs per systems seem to be located preferably at the end of tidal tails. Not much structure elsewhere in the same 


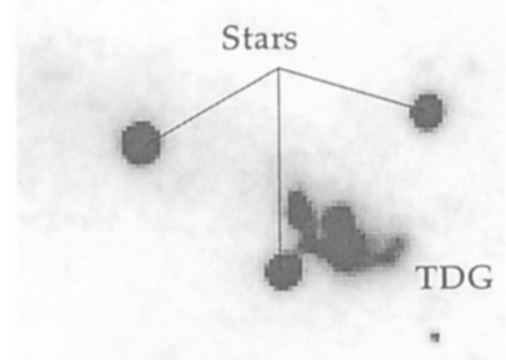

Figure 2. $\quad B$-band image of the TDG in AM 1159-530.

tail. Examples: NGC 7252W (Knierman et al. 2001), AM 1159-530 (Weilbacher \& Duc 2001).

2. SSCs/GCs: Lots of star clusters are seen along the tidal tail, age and association not well restricted. Example: Western tail of NGC 3256 (Knierman et al. 2001).

3. Extended knots: A chain of extended knots is seen along the tidal tail. They are not resolved into HII regions, and therefore represent an intermediate case. Example: Both tails of AM 1353-272 (Fig. 1).

The creation of TDGs (case 1) has been successfully modeled dynamically, although the difference between stellar (Barnes \& Hernquist 1992) and gaseous (Elmegreen et al. 1993) condensations is observationally not as pronounced as in the models. The creation of SSCs or GCs (case 2) in tidal tails is not understood, as it is generally thought that high gas densities are necessary to produce such dense clusters. It is not clear how the intermediate case (3) relates to the other phenomena. Is it possible that some of these knots merge to form a massive TDG, or are they dispersed quickly by the tidal field?

\section{Star formation and morphology of TDGs}

TDGs do actively form stars and contain molecular gas, but what are the morphological properties of this star formation? From optical observations, especially highly resolved HST images (Knierman et al. 2001), one can classify two types of TDGs, from the viewpoint of star formation and HII regions:

a. Dense and knotty: Several clusters or HII regions are visible in TDGs. Examples: AM 1159-530 (Fig. 2), NGC 7252W.

b. Large and LSB: A few clusters are seen in an extended very low surface brightness object, where the association of the clusters to the objects may also be accidental. Example: NGC 7252E.

Type a shows internal kinematics and therefore represents true TDGs, while this has not been shown without ambiguity for type $b$, where more and deeper 
spectroscopic data is needed to prove decoupled dynamics. Although the morphology of these two types is quite different, their colors are similarly blue, and their spectra both show strong emission lines, implying comparable star formation activity. A possible evolutionary connection between these two "classes" of TDGs must remain speculative for the moment.

\section{Conclusions}

We see 3 modes of young structure in tidal tails, and two types of TDGs. It is currently unknown what effects are responsible for these different structures. Especially the possibility of forming GCs/SSCs in tidal tails is uncertain. New dynamical models are needed which provide high enough resolution to resolve small scale structures along the tails and in the TDGs themselves, and possibly include SF and feedback to study how SF takes place in TDGs, and what repercussions stellar winds and supernovae have on the evolution of TDGs.

From the observational point of view spectrophotometric analysis of TDG (candidates) is needed to confirm more 'knots' as TDGs. Further one needs to constrain total and stellar mass, and the burst properties and possible fading using a wider wavelength range. Integral field spectroscopy is needed to measure internal kinematics of TDGs and assess their stability.

Acknowledgements. PMW thanks the Astronomische Gesellschaft and the organizers for generous support and J.E. Hibbard for stimulating discussions.

\section{References}

Barnes, J.E., Hernquist, L. 1992, Nature, 360, 715

Duc, P.-A., Brinks, E., Springel, V., Pichardo, B., Weilbacher, P., Mirabel, I.F. 2000, AJ, 120, 1238

Duc, P.-A., Brinks, E., Wink, J.E. \& Mirabel, I.F. 1997, A\&A, 326, 537

Elmegreen, B.G., Kaufman, M., Thomasson, M. 1993, ApJ, 412, 90

Hibbard, J.E., Mihos, J.C. 1995, AJ, 110, 140

Hibbard, J.E. 2001, Meeting on Tidal Dwarf Galaxies, http://www.uni-sw.gwdg.de/ paduc/TDGmeet/hibbard/Saclay01/

Knierman, K.A., Gallagher, S.C., Charlton, J.C., Hunsberger, S.D., Whitmore, B., Kundu, A, Hibbard, J. E., Zaritsky, D. 2001, AJ, submitted

Lisenfeld, U., Braine, J., Vallejo, O. Duc, P.-A., Leon, S., Brinks, E., Charmandaris, V. 2001 in Modes of Star Formation and the Origin of Field Population, ed. E.K. Grebel \& W. Brandner (astro-ph/0101319)

Weilbacher, P.M., Duc, P.-A., Fritze-v.Alvensleben, U., Martin, P., Fricke, K.J. 2000, A\&A, 358, 819

Weilbacher, P. \& Fritze-v.Alvensleben, U. 2001 in Dwarf Galaxies and their Environment, ed. K.S. de Boer, R.-J. Dettmar, U. Klein, in press (astro$\mathrm{ph} / 0105272)$

Weilbacher, P. \& Duc, P.-A. 2001 in Dwarf Galaxies and their Environment, ed. K.S. de Boer, R.-J. Dettmar, U. Klein, in press (astro-ph/0105275) 


\section{Discussion}

B. Elmegreen: There are two different processes by which tidal tails can make dwarf galaxies, one in which the tidal arm is unstable to collapse along its length, making beads of clusters as in a spiral arm, and another is the formation of a giant HI cloud at the end of the arm as a result of movement of the entire outer HI disk. In addition, all the clusters initially at intermediate radius in the pre-collision galaxy will end up in the tidal arm too, because of the tidal motions.

P. Weilbacher: I agree that in principle one can explain how these different structures can be formed in tidal tails. But simulations only show one of these effects at a time, while in real interacting systems, like e.g. NGC 7252 , we see two or more different "types" of TDGs. Models can currently not explain why we see which structures in which tails created during which kind of encounter geometry. That's why I think we need high resolution dynamical models.

\section{S. Sakhibov: What is the range of metallicities in studied objects?}

P. Weilbacher: TDGs have metallicities of $Z \approx 1 / 3 Z_{\odot}$ or $\log O / H \approx 8.5$.

T. Armandroff: The galaxies of the Local Group and nearby groups define a relation between absolute magnitude and mean metallicity, with very few outliers. Are you using this relation as a diagnostic for tidal dwarfs? And can you use this relation to establish how common surviving tidal dwarfs are?

P. Weilbacher: Yes, the known (candidates of) TDGs have a more or less constant metallicity of the value cited before, and do not follow the luminositymetallicity relation. This is used to discriminate TDGs from possible preexisting dwarfs in interacting systems, and should in principle allow to see if a dwarf galaxy has tidal origin.

E. Telles: What is the fate of these condensations formed in these tails predicted by the models? Will they fall back into the system? Also, it is unlikely that this is a major mechanism for dwarf galaxy formation because dwarf galaxies are metal poor and TDGs are not. And we (Telles \& Terlevich 1995) find no evidence for giant companions around star forming dwarfs such as HII galaxies.

P. Weilbacher: In the general models of Barnes \& Hernquist (1992) and

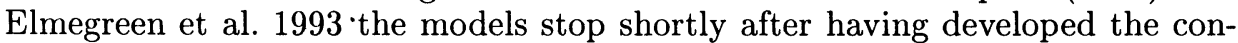
densations. But in the modelling of NGC 7252 (Hibbard \& Mihos 1995) it is noted that the outer half of the tails can escape or reach orbits of several Gyrs around the central merger.

About the comment: We do not expect that the major number of dwarf galaxies is formed as TDGs. But your sample and other samples are preselected to include only galaxies that show the faint emission line [OIII]4363. It is therefore preselected for metal poor galaxies, and the missing TDGs might be a selection effect. We also expect TDGs not to keep up their current SFR for a long time, but instead they could fade by several magnitudes within one Gyr and then they will not be detected in surveys, especially emission line surveys will miss them. 